\title{
Path Loss Measurement for GSM Terrains in Lagos State, Nigeria
}

\author{
N. O. Salau \\ Department of Electronic and \\ Computer Engineering, \\ Lagos State University, \\ Epe, Lagos. Nigeria.
}

\author{
O. O. Shoewu \\ Department of Electronic and \\ Computer Engineering, \\ Lagos State University, \\ Epe, Lagos. Nigeria
}

\author{
A. O. Ogunlewe \\ Department of Electonic and \\ Computer Engineering, \\ Lagos State University, \\ Epe, Lagos, Nigeria
}

\begin{abstract}
This paper presents report of research work on comparative analysis of Pathloss measurements based on terrain differences, frequencies of operation and distance between Base Transceiver Station (BTS) and Mobile Station (MS). Lagos state G.S.M terrains with $2 \mathrm{G}(900 \mathrm{MHz} / 1800 \mathrm{MHz})$ and $3 \mathrm{G}(2100 \mathrm{MHz})$ frequencies of operation were put under investigation. Field data were collected through drive tests from twelve (12) different sites using TEMS software in the chosen environments over a range of distance between 0.5$10 \mathrm{Km}$ from Base station (BS) to Mobile station (MS) with measurement taken at $0.5 \mathrm{Km}$ intervals for a period of one year (52 weeks). Strategically Lagos-Island $\left(\mathrm{N6}^{0} 27.4832{ }^{\prime} \mathrm{E}^{0}\right.$ 23.5453'), Surulere (N6 ${ }^{0} 33.3844^{\prime}$ E3 ${ }^{0} 20.9407^{\prime}$ '), Lekki-Oniru $\left(\mathrm{N}^{0}{ }^{2} 26.6661{ }^{\prime} \mathrm{E}^{0}{ }^{2} 28.7463\right.$ ') and Agbede-Ikorodu (N6 ${ }^{0}$ $39.9250^{\prime} \mathrm{E}^{0}{ }^{2} 29.0363$ ') were chosen as dense-urban (DU), urban (UR), sub-urban (SU) and non-urban (NU) G.S.M terrains respectively. In this research work Rxlev/RSSI and Pathloss were measured in all areas of investigation and empirical Cost231-Hata model was used for pathloss calculation of field data, and its results were compared with Pathloss measured through drive tests. The measured pathloss, when compared with empirical values from the COST 231 theoretical models, showed a close agreement with the pathloss predicted by the COST231- Hata model in terms of mean square error analysis. During our findings it was revealed that Pathloss increases with distance between BTS and MS, Pathloss also increases with frequency of operation; hence $3 \mathrm{G}$ networks experience more pathloss than $2 \mathrm{G}$ and from further analysis it was observed that Pathloss increases from Non-Urban to Sub-Urban to Urban and Dense-Urban respectively when compared using different terrain characteristics.
\end{abstract}

\section{General Terms}

Comparative analysis of pathloss measurements

\section{Keywords}

GSM, RSS, RSSI, Terrain and Pathloss

\section{INTRODUCTION}

Path loss can be defined as reduction in power density of electromagnetic wave as it propagates through space ${ }^{[13]}$, i.e. it is the difference (in $\mathrm{dB}$ ) between the transmitted power and the received power. ${ }^{[11][12]}$

$\mathrm{P}_{\mathrm{L}}(\mathrm{dB})=\mathrm{P}_{\mathrm{T}}(\mathrm{dB})-\mathrm{P}_{\mathrm{R}}(\mathrm{dB})$

In other words it can be viewed as the loss which the signal experiences as it radiates from transmitter to receiver, meaning the difference in the signal strengths from transmitter (source) to receiver (destination) antenna is termed Path loss. [14]

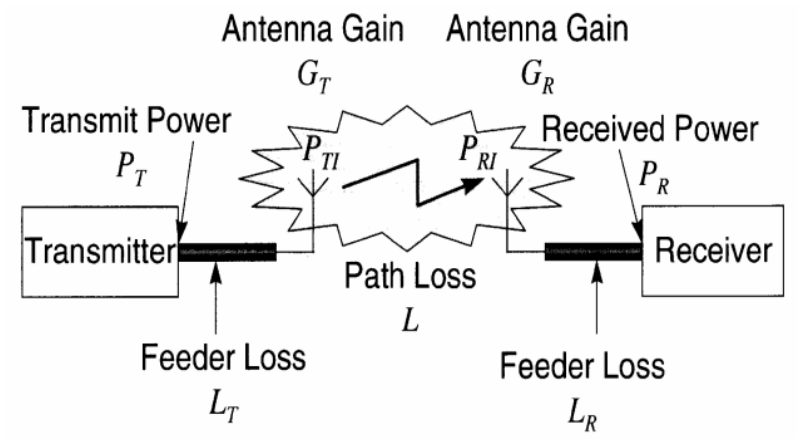

Fig 1: Path loss indications on a communication link ${ }^{[11]}$

\section{PROBLEM STATEMENT}

Comparative analysis of pathloss prediction models for Urban and Sub-Urban environs under 900 and $1800 \mathrm{MHz}(2 \mathrm{G})$ frequencies of operation have been done by [15][16], comparison between $1800 /$ WCMDA i.e. $(2 \mathrm{G} / 3 \mathrm{G})$ frequencies of operation was also carried out by [17], all in effort to validate the applicability of known empirical models in 1 0r 2 different G.S.M environs (Urban only or Urban/Sub-urban) or with research consideration to $900 \mathrm{MHz}^{[15]}$ only or $900 / 1800 \mathrm{MHz}^{[16]}$ or $1800 \mathrm{MHz}^{[3]}$ only or $1800 / 2100 \mathrm{MHz}^{[17]}$ frequencies of operation. Extensive analysis was also carried out by [1] on five-3G parameters still using one frequency of operation $(2100 \mathrm{MHz})$ in one environment (sub-urban). ${ }^{[1]}$

This research work has enhanced these former research works by considering three frequencies of operation i.e. 900,1800 and $2100 \mathrm{MHz}$ available in $2 \mathrm{G}$ and $3 \mathrm{G}$ bands and has deviated from the traditional comparison based on propagation models but utilized the conclusions of past projects ${ }^{[1][3]}$ which suggested that Cost 231-Hata model showed the best performance in Lagos environments, hence adopted as a reference model. Cost 231 model is also chosen because of its peculiarity which makes it useful for predicting signal strength in all environments ${ }^{[5]}{ }^{[7]}$, and its frequency range that extends to $2000 \mathrm{MHz}^{[2]}$, and its incorporated signal strength prediction of up to $20 \mathrm{~km}$ from transmitter to receiver with transmitter antenna height ranging from $30 \mathrm{~m}$ to $200 \mathrm{~m}$ and receiver antenna height ranging from $1 \mathrm{~m}$ to $10 \mathrm{~m}^{[5]}[6]$.

Therefore our pathloss comparison is based on terrain differences using four environments (NU, SU, UR, DU), frequencies of operations $900 / 1800 / 2100 \mathrm{MHz}$ and distance between BTS and MS respectively.

\section{INVESTIGATED AREAS 3.1 Propagation Medium}

The propagation medium can be classified into several propagation environments such as: 
* Non-Urban / Rural terrain

* Sub-Urban terrain

* Micro- Urban terrain

* Peri- Urban terrain

* Ex-Urban terrain

* Urban terrain

* Dense Urban terrain

For the purpose of this project only four terrains: Non-Urban, Sub-Urban, Urban and Dense-Urban are chosen as study areas.

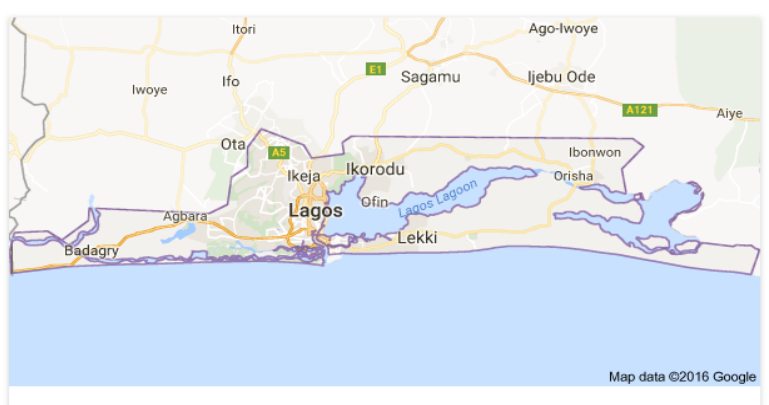

Lagos

Fig 2: Map of Lagos State from Google

\subsection{Methodology}

The method adopted for data collection in this research work was drive testing approach using the popular Ericson product, now owned by ASCOM, the investigation software called the TEMS. Data were collected carefully and analyzed with TEMS version 13 and MapInfo version 11.

The pathloss was evaluated with the use of COST 231Hata Model and its results were used for comparison based on terrain difference, frequency of operation and distance between BTS and MS.

The tools that were used to carry out the drive test for the data collection for this research work include:

- TEMS Mobiles(W995)

- GPS

- Test Sims-fromProvider

- TEMS software Version 13 with related DONGLE (Secunity Key)

- Mapinfo software version 11

- Database of the sites

- Country vector and Cellfile and other required Tabfiles

- Power Inverter - car supported inverter used to power the Laptop

- Laptop

- $\mathrm{Cab}$

\subsection{Experimental Setup of Drive Test}

- All the components (GPS, TEMs phones) except the dongle were connected appropriately to the laptop via the Hub (for convenience) to take simultaneous reading of $2 \mathrm{G}$ and $3 \mathrm{G}$ in both idle and active mode. See figure 9 .

- Then connect the laptop power cord to car supported inverter for constant electricity supply, power ON the laptop after which the dongle will be connected to it through one of its USB (Universal Serial Bus) ports

- The dongle connected to the laptop gives a license to the TEMS interface on the system, without which the TEMS phones can be viewed and accessed. The dongle also allows accessibility of GPS readings on TEMS interface.

- Next step is to launch the TEMS and commence investigation by pressing the button RECORD on the menu, while the cab moves at a speed not more that $40 \mathrm{Km} / \mathrm{h}$.

- $\quad$ TEMS has audio capability which helps to quickly detect any disconnected component. This is achieved with the encrypted voice in the TEMS which loudly notifies the DT technician if there is any changes in the call setup or disconnection. ${ }^{[1]}$

- After the successful connection of these tools, the next step is to get the car moving and the drive test commences.

- The drive testing lasted for about one and a half hour for each of the 12 sites in each environments investigated.

- Measurements were taken twice in a day for a period of one year and mean average values of received signal strength obtained for project analysis.

\subsection{Flow chart of Drive test}

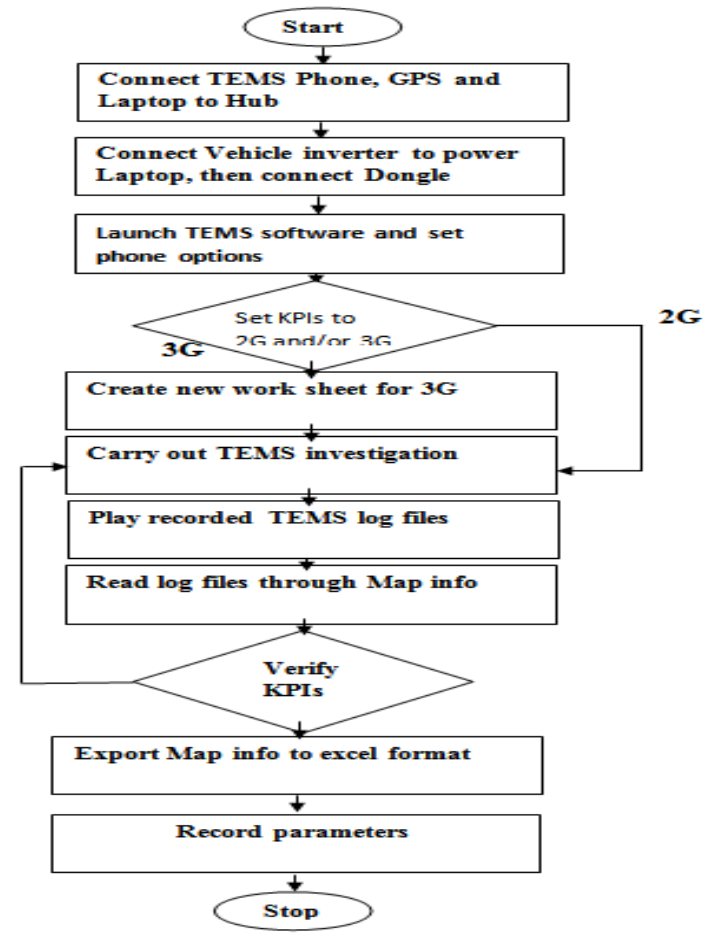

Fig 3: Flow chart of Drive Test 


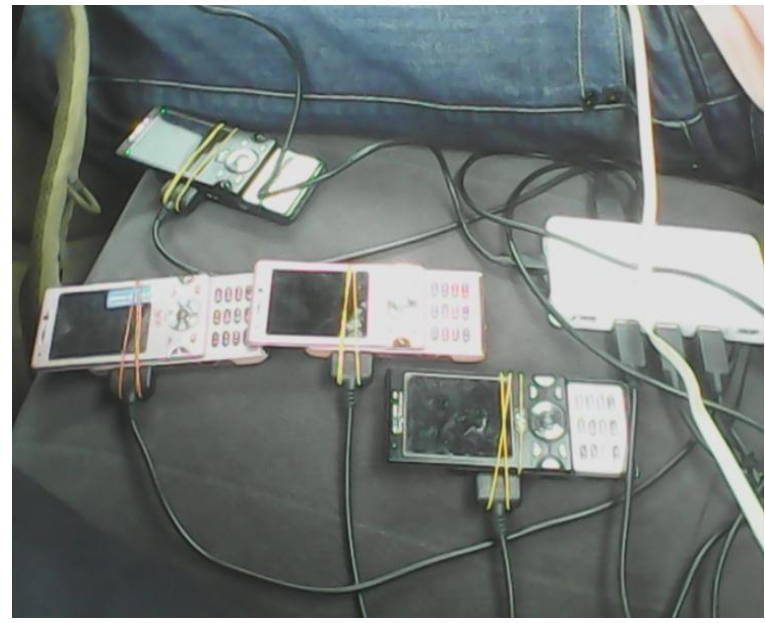

Fig 4: Drive Test TEMS Phones

\subsection{Measured Parameters in 2G Networks}

Quality of GSM/EDGE 2G 900/1800 MHz coverage is described basically by two indicators (KPIs); according to ECC Report $118(2008)^{[8]}$ these are:

\section{- Receive Level}

RxLev -this is the received signal strength on serving cell, measured respectively on all slots RxLevFull and on a subset of slots RxLevSub. RxLevel is received power level at MS (maximum RxLevel measured by MS is $\left( \pm\right.$ ) $-40 \mathrm{dBm}^{[4]}$

\section{- $\quad$ Receive Quality}

RxQual -this is the received signal quality on serving cell, measured respectively on all slots (RxQualFull) and on a subset of the slots (RxQualSub). ${ }^{[4]}$

Received signal quality level, are measured based on BER (bit error rate). The value is between $0-7$, the lower the better.

\section{- (C) Speech Quality Index (SQI)}

SQI is a performance metric for voice quality in telecommunication.

It is specific only to the TEMS family of drive testing/field testing tools. SQI aims to provide a reasonable estimate of the voice quality, as perceived by a human ear. ${ }^{[1]}$

\subsection{Measured Parameters in 3G Networks}

Quality of UMTS 3G $2100 \mathrm{MHz}$ coverage is described basically by three indicators, according to the ECC Report $103(2007){ }^{[8]}$ these are

\section{- $\quad$ Received Signal Code Power (RSCP)}

$\mathrm{RSCP}$ is the received power on one code measured on the pilot bits of the P-CPICH (Primary Common Pilot Channel).

- $\quad$ Received Signal Strength Indicator

RSSI is the wideband received power within the relevant channel bandwidth; it is the measure of received signal strength in $3 \mathrm{G}$ domain.

\section{- Ec/No Service Quality}

$\mathrm{Ec} / \mathrm{NO}$ is the ratio of received pilot energy, $E c$, to the total received energy or the total power spectral density, $I O$.The received energy per chip, $E c$, divided by the power density in the band. The Ec/NO is identical to RSCP/RSSI ${ }^{[8]}$. Measured in decibel; $\mathrm{dB}^{[1]}$.

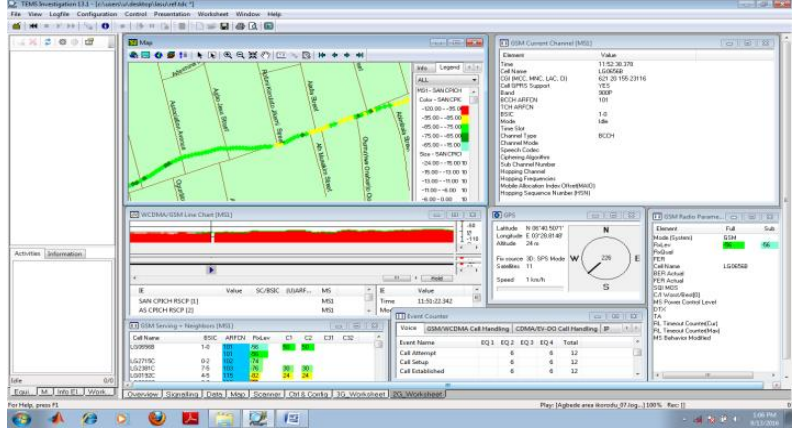

Fig 5: TEMS Investigation Interface for NonUrban(Agbede-Ikorodu)

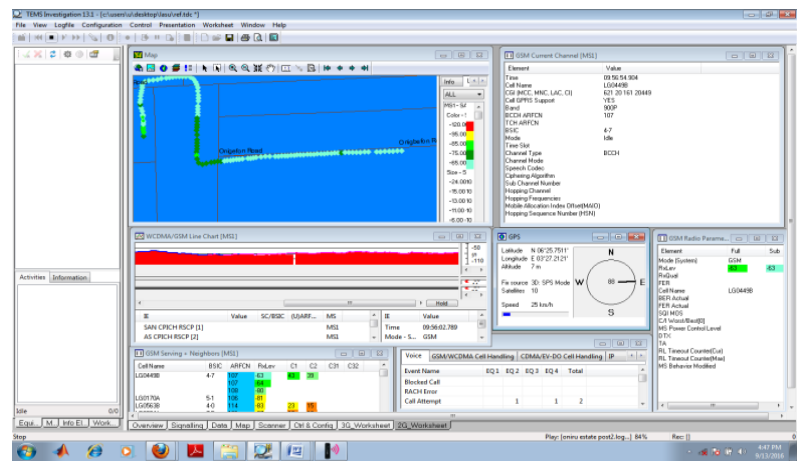

Fig 6: TEMS Investigation Interface for SubUrban(Lekki-Oniru) environ

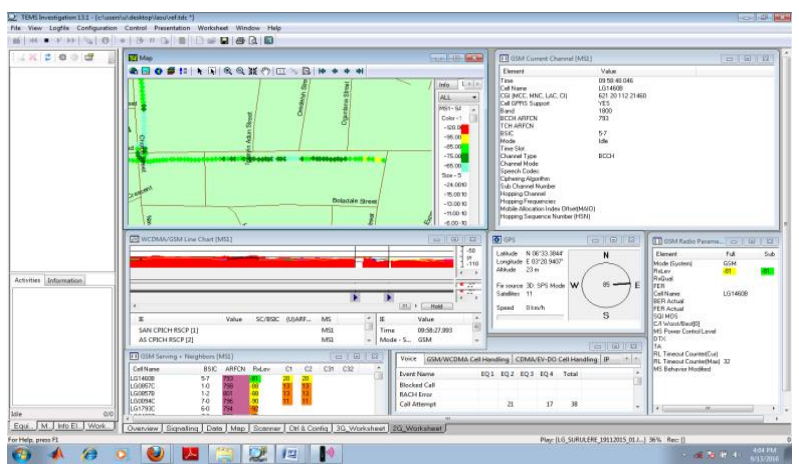

Fig 7: TEMS Investigation Interface for Urban(Surulere) Environ

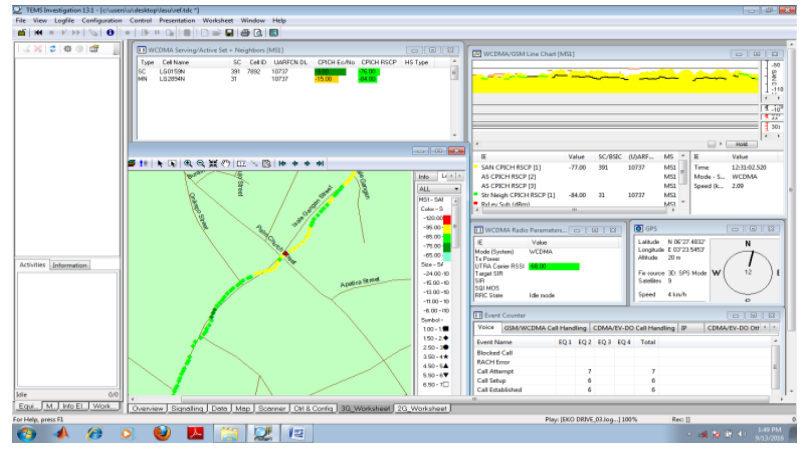

Fig 8: TEMS Investigation Interface for DenseUrban(Lagos-Island) Environ 
4. DATA ANALYSIS OF PATHLOSS

Table 1. G.S.M Environments and R.F Parameters

\begin{tabular}{|l|l|l|}
\hline Environment & BTS Power & BTS Antenna Height \\
\hline $\begin{array}{l}\text { Non } \\
\text { urban(Rural) }\end{array}$ & $43 \mathrm{dBm}$ & $45 \mathrm{~m}$ \\
\hline Urban & $38 \mathrm{dBm}$ & $35 \mathrm{~m}$ \\
\hline Suburban & $43 \mathrm{dBm}$ & $40 \mathrm{~m}$ \\
\hline Dense-Urban & $36 \mathrm{dBm}$ & $30 \mathrm{~m}$ \\
\hline
\end{tabular}

Where Connection loss $=4.3 \mathrm{dBi}$, Feeder loss $=0.3 \mathrm{dBi}$, Duplexer loss $=2.1 \mathrm{dBi}$, Antenna Gain $=2.1 \mathrm{dBi}$, BTS antenna Gain=14dBi

Considering the distance $\mathrm{d}(\mathrm{km})$ intervals used for drive test, the measured path loss $\mathrm{PL}_{\mathrm{m}}(\mathrm{dB})$ for each terrain can be found using equations given by Rappaport (2002) $)^{[9]}$ and Seybold $(2005)^{[10]}$ as:

$\mathrm{PL}_{\mathrm{m}}(\mathrm{dB})=\mathrm{EIRP}_{\mathrm{t}}(\mathrm{dBm})-\mathrm{P}_{\mathrm{r}}(\mathrm{dBm})$

Where $\mathrm{EIRP}_{\mathrm{t}}=$ effective isotropic radiated power in $\mathrm{dBm}$ and $\mathrm{P}_{\mathrm{r}}=$ mean power received in $\mathrm{dBm}$.

The effective isotropic radiated power $\operatorname{EIRP}_{t}(\mathrm{dBm})$ is given as:

$\mathrm{EIRP}_{\mathrm{t}}=\mathrm{P}_{\mathrm{BTS}}+\mathrm{G}_{\mathrm{BTS}}+\mathrm{G}_{\mathrm{MS}}-\mathrm{L}_{\mathrm{FC}}-\mathrm{L}_{\mathrm{AB}}-\mathrm{L}_{\mathrm{CF}} \ldots$

Where $\mathrm{P}_{\mathrm{BTS}}=\mathrm{BTS}$ power $(\mathrm{dBm})$,

$\mathrm{G}_{\mathrm{BTS}}=\mathrm{BTS}$ antenna gain (dBi),

$\mathrm{G}_{\mathrm{MS}}=\mathrm{MS}$ antenna gain $(\mathrm{dBi})$,

$\mathrm{L}_{\mathrm{FC}}=$ feeder cable and connector loss $(\mathrm{dB})$,

$\mathrm{L}_{\mathrm{AB}}=$ antenna body loss $(\mathrm{dB})$ and $\mathrm{L}_{\mathrm{CF}}=$ combiner and filter loss (dB).

Substituting the values in TABLE 1 into equation (3), we calculated $\mathrm{EIRP}_{\mathrm{t}}$ thus:

\section{FOR NON - URBAN ENVIRONMENT}

$\mathrm{EIRP}_{\mathrm{t}}=\mathrm{PBTS}+\mathrm{GBTS}+\mathrm{GMS}-\mathrm{LFC}-\mathrm{LAB}-\mathrm{LCF}$

$$
\begin{aligned}
& =43+14+2 \cdot 1-0 \cdot 3-2 \cdot 1-4 \cdot 3 \\
& =52 \cdot 4 \mathrm{~dB}
\end{aligned}
$$

\section{FOR SUB - URBAN ENVIRONMENT}

$$
\begin{aligned}
\mathrm{EIRP}_{\mathrm{t}} & =\mathrm{PBTS}+\mathrm{GBTS}+\mathrm{GMS}-\mathrm{LFC}-\mathrm{LAB}-\mathrm{LCF} \\
& =43+14+2.1-0.3-2.1-4.3 \\
& =52.4 \mathrm{~dB}
\end{aligned}
$$

\section{FOR URBAN ENVIRONMENT}

$$
\begin{aligned}
\mathrm{EIRP}_{\mathrm{t}} & =\mathrm{PBTS}+\mathrm{GBTS}+\mathrm{GMS}-\mathrm{LFC}-\mathrm{LAB}-\mathrm{LCF} \\
& =38+14+2.1-0.3-2.1-4.3 \\
& =47.4 \mathrm{~dB}
\end{aligned}
$$

FOR DENSE - URBAN ENVIRONMENT

$\mathrm{EIRP}_{\mathrm{t}}=\mathrm{PBTS}+\mathrm{GBTS}+\mathrm{GMS}-\mathrm{LFC}-\mathrm{LAB}-\mathrm{LCF}$

$$
=36+14+2 \cdot 1-0 \cdot 3-2 \cdot 1-4 \cdot 3
$$

$$
=45.4 \mathrm{~dB}
$$

The EIRPt values calculated above were further inserted into equation (2) and tables (2-4) of measured Pathloss $\left(\mathrm{PL}_{\mathrm{m}}\right)$ were compiled

Table 2. Overall Average Measured PL for 2G-900 MHz

\begin{tabular}{|l|l|l|l|l|}
\hline Distance & NU & SU & UR & DU \\
\hline 0.5 & 95.07 & 104.48 & 99.32 & 87.82 \\
\hline 1 & 97.48 & 107.48 & 101.4 & 88.82 \\
\hline 1.5 & 103.98 & 113.98 & 105.48 & 91.57 \\
\hline 2 & 107.07 & 118.57 & 109.98 & 96.82 \\
\hline 2.5 & 114.15 & 124.73 & 115.07 & 97.98 \\
\hline 3 & 115.9 & 126.57 & 117.57 & 100.73 \\
\hline 3.5 & 117.32 & 129.4 & 121.9 & 106.65 \\
\hline 4 & 123.82 & 131.15 & 124.9 & 108.15 \\
\hline 4.5 & 124.98 & 135.48 & 127.57 & 110.82 \\
\hline 5 & 125.65 & 138.57 & 131.07 & 116.07 \\
\hline 5.5 & 132.9 & 142.73 & 135.15 & 119.07 \\
\hline 6 & 135.82 & 146.15 & 139.23 & 123.9 \\
\hline 6.5 & 141.65 & 148.57 & 141.15 & 126.73 \\
\hline 7 & 143.57 & 150.9 & 145.65 & 128.4 \\
\hline 7.5 & 146.07 & 152.57 & 147.57 & 134.9 \\
\hline 8 & 147.65 & 153.82 & 149.9 & 136.98 \\
\hline 8.5 & 151.23 & 156.57 & 151.4 & 140.48 \\
\hline 9 & 152.73 & 158.4 & 151.65 & 144.73 \\
\hline 9.5 & 154.48 & 161.82 & 153.23 & 146.98 \\
\hline 10 & 159.15 & 164.32 & 159.07 & 151.15 \\
\hline
\end{tabular}

Table 3. Overall Average Measured PL for 2G-1800 MHz

\begin{tabular}{|l|l|l|l|l|}
\hline Distance & NU & SU & UR & DU \\
\hline 0.5 & 107.82 & 109.23 & 103.9 & 102.23 \\
\hline 1 & 112.82 & 113.82 & 106.73 & 106.15 \\
\hline 1.5 & 116.07 & 116.65 & 110.48 & 109.48 \\
\hline 2 & 118.32 & 120.9 & 113.4 & 112.15 \\
\hline 2.5 & 122.57 & 122.9 & 116.48 & 115.15 \\
\hline 3 & 126.48 & 126.4 & 117.4 & 117.82 \\
\hline 3.5 & 128.48 & 128.82 & 120.9 & 120.65 \\
\hline 4 & 130.57 & 132.07 & 121.9 & 123.07 \\
\hline 4.5 & 133.73 & 134.32 & 123.98 & 126.48 \\
\hline 5 & 136.23 & 136.73 & 128.4 & 128.73 \\
\hline 5.5 & 139.15 & 138.4 & 130.73 & 131.48 \\
\hline 6 & 141.57 & 141.4 & 134.4 & 134.57 \\
\hline 6.5 & 143.9 & 144.73 & 137.23 & 135.98 \\
\hline 7 & 146.65 & 146.15 & 140.9 & 139.32 \\
\hline 7.5 & 148.9 & 149.23 & 143.82 & 142.4 \\
\hline
\end{tabular}




\begin{tabular}{|l|l|l|l|l|}
\hline 8 & 151.48 & 152.65 & 146.9 & 145.32 \\
\hline 8.5 & 153.07 & 154.82 & 149.98 & 148.48 \\
\hline 9 & 156.32 & 157.07 & 151.73 & 151.15 \\
\hline 9.5 & 158.73 & 160.32 & 153.23 & 152.9 \\
\hline 10 & 162.73 & 163.15 & 157.9 & 156.57 \\
\hline
\end{tabular}

Table 4. Overall Average Measured PL for 3G-2100 MHz

\begin{tabular}{|l|l|l|l|l|}
\hline Distance & NU & SU & UR & DU \\
\hline 0.5 & 96.57 & 105.98 & 101.15 & 89.32 \\
\hline 1 & 99.98 & 111.65 & 107.82 & 90.57 \\
\hline 1.5 & 106.07 & 118.48 & 111.07 & 94.48 \\
\hline 2 & 109.65 & 124.23 & 113.48 & 98.73 \\
\hline 2.5 & 116.73 & 129.07 & 124.65 & 100.07 \\
\hline 3 & 118.23 & 130.57 & 126.23 & 103.4 \\
\hline 3.5 & 120.23 & 131.48 & 127.07 & 108.65 \\
\hline 4 & 125.57 & 133.4 & 127.32 & 110.73 \\
\hline 4.5 & 127.32 & 138.4 & 129.82 & 114.15 \\
\hline 5 & 129.32 & 143.9 & 132.23 & 118.73 \\
\hline 5.5 & 135.32 & 150.23 & 136.73 & 121.73 \\
\hline 6 & 138.73 & 150.98 & 139.82 & 126.4 \\
\hline 6.5 & 143.48 & 152.57 & 141.15 & 130.07 \\
\hline 7 & 145.32 & 154.57 & 147.4 & 132.15 \\
\hline 7.5 & 147.98 & 156.4 & 151.23 & 138.4 \\
\hline 8 & 149.82 & 157.07 & 151.98 & 140.48 \\
\hline 8.5 & 152.07 & 160.15 & 156.65 & 142.82 \\
\hline 9 & 154.23 & 161.4 & 155.4 & 147.48 \\
\hline 9.5 & 157.15 & 163.82 & 157.15 & 151.4 \\
\hline 10 & 157.44 & 162.9 & 157.92 & 151.36 \\
\hline & & & & \\
\hline & & & & \\
\hline & & & & \\
\hline
\end{tabular}

\subsection{Data analysis of Calculated Pathloss using Cost231 Model}

Calculations of Empirical Pathloss were achieved using Cost 231 pathloss model equation given as:

$\mathrm{P}_{\mathrm{L}}(\mathrm{dB})=46.3+33.9 \log _{10} f_{c}-13.82 \log _{10}\left(h_{t}\right)-\mathrm{a}\left(h_{r}\right)+[44.9-6.551 \mathrm{lo}$ $\left.\mathrm{g} 10 h_{t}\right] \log _{10} d+C$.

Where:

$\mathrm{C}=0 \mathrm{~dB}$, for suburban areas or open environments and $3 \mathrm{~dB}$ for Urban environment ${ }^{[1][3]}$

$\left(h_{r}\right)=$ mobile station antenna height correction factor is defined as:

$\mathrm{a}\left(\mathrm{h}_{\mathrm{r}}\right)=\left(1.11 \log _{10} f_{c}-0.7\right) h_{r}-\left(1.5 \log 10 f_{c}-0.8\right)$, for suburban or rural areas ${ }^{[1][3]}$

$\mathrm{a}\left(\mathrm{h}_{\mathrm{r}}\right)=3.20\left[\log _{10}(11.75 \mathrm{hr})\right]-4.97$ for $\mathrm{f}>400 \mathrm{MHz}$ for Urban environment ${ }^{[3]}$

Substituting the $f_{c}$ as appropriate: 900 or 1800 or 2100 and $\mathrm{a}\left(\mathrm{h}_{\mathrm{r}}\right)$ as given above and $\mathrm{h}_{\mathrm{t}}$ from Table 1 above,

where $h_{r}($ Height of MS $)=3 \mathrm{~m}$, and d is distance between BTS and MS.(0.5-10Km), we compiled the tables (5-7) thus:
Table 5. Overall Average Calculated PL for 2G-900 MHz

\begin{tabular}{|l|l|l|l|l|}
\hline Distance & NU & SU & UR & DU \\
\hline 0.5 & 109.5 & 110.11 & 114.95 & 115.74 \\
\hline 1 & 119.76 & 120.47 & 125.42 & 126.35 \\
\hline 1.5 & 125.76 & 126.53 & 131.55 & 132.55 \\
\hline 2 & 130.02 & 130.83 & 135.89 & 136.95 \\
\hline 2.5 & 133.32 & 134.16 & 139.26 & 140.36 \\
\hline 3 & 136.02 & 136.88 & 142.02 & 143.15 \\
\hline 3.5 & 138.3 & 139.19 & 144.35 & 145.51 \\
\hline 4 & 140.27 & 141.18 & 146.36 & 147.55 \\
\hline 4.5 & 142.02 & 142.94 & 148.14 & 149.35 \\
\hline 5 & 143.58 & 144.52 & 149.73 & 150.97 \\
\hline 5.5 & 144.99 & 145.94 & 151.17 & 152.42 \\
\hline 6 & 146.27 & 147.24 & 152.49 & 153.76 \\
\hline 6.5 & 147.46 & 148.44 & 153.7 & 154.98 \\
\hline 7 & 148.55 & 149.54 & 154.82 & 156.11 \\
\hline 7.5 & 149.58 & 150.58 & 155.86 & 157.17 \\
\hline 8 & 150.53 & 151.54 & 156.84 & 158.16 \\
\hline 8.5 & 151.43 & 152.45 & 157.75 & 159.08 \\
\hline 9.5 & 152.27 & 153.3 & 158.61 & 159.96 \\
\hline 10 & 153.07 & 154.11 & 159.43 & 160.79 \\
\hline 5 & 154.87 & 160.21 & 161.57 \\
\hline
\end{tabular}

Table 6. Overall Average Calculated PL for 2G-1800 MHz

\begin{tabular}{|l|l|l|l|l|}
\hline Distance & NU & SU & UR & DU \\
\hline 0.5 & 119.19 & 119.79 & 125.15 & 125.95 \\
\hline 1 & 129.44 & 130.15 & 135.62 & 136.55 \\
\hline 1.5 & 135.44 & 136.21 & 141.75 & 142.75 \\
\hline 2 & 139.7 & 140.51 & 146.1 & 147.15 \\
\hline 2.5 & 143 & 143.84 & 149.47 & 150.57 \\
\hline 3 & 145.7 & 146.57 & 152.22 & 153.36 \\
\hline 3.5 & 147.98 & 148.87 & 154.55 & 155.71 \\
\hline 4 & 149.96 & 150.86 & 156.57 & 157.76 \\
\hline 4.5 & 151.7 & 152.62 & 158.35 & 159.56 \\
\hline 5 & 153.26 & 154.2 & 159.94 & 161.17 \\
\hline 5.5 & 154.67 & 155.62 & 161.38 & 162.63 \\
\hline 6 & 155.95 & 156.92 & 162.69 & 163.96 \\
\hline 6.5 & 157.14 & 158.12 & 163.9 & 165.18 \\
\hline 7 & 158.24 & 159.23 & 165.02 & 166.32 \\
\hline 7.5 & 159.26 & 160.26 & 166.07 & 167.37 \\
\hline 8 & 160.21 & 161.22 & 167.04 & 168.36 \\
\hline 8.5 & 161.11 & 162.13 & 167.96 & 169.29 \\
\hline 9 & 161.95 & 162.98 & 168.82 & 170.16 \\
\hline 9.5 & 162.75 & 163.79 & 169.64 & 170.99 \\
\hline & & & & \\
\hline & & & & \\
\hline & & & & \\
\hline
\end{tabular}




\begin{tabular}{|l|l|l|l|l|}
\hline 10 & 163.51 & 164.56 & 170.41 & 171.77 \\
\hline
\end{tabular}

Table 7. Overall Average Calculated PL for 3G-2100 MHz

\begin{tabular}{|l|l|l|l|l|}
\hline Distance & NU & SU & UR & DU \\
\hline 0.5 & 121.34 & 121.94 & 127.42 & 128.22 \\
\hline 1 & 131.6 & 132.3 & 137.89 & 138.82 \\
\hline 1.5 & 137.59 & 138.36 & 144.02 & 145.02 \\
\hline 2 & 141.85 & 142.66 & 148.37 & 149.42 \\
\hline 2.5 & 145.15 & 145.99 & 151.74 & 152.84 \\
\hline 3 & 147.85 & 148.72 & 154.49 & 155.63 \\
\hline 3.5 & 150.13 & 151.02 & 156.82 & 157.98 \\
\hline 4 & 152.11 & 153.02 & 158.84 & 160.03 \\
\hline 4.5 & 153.85 & 154.78 & 160.62 & 161.83 \\
\hline 5 & 155.41 & 156.35 & 162.21 & 163.44 \\
\hline 5.5 & 156.82 & 157.78 & 163.65 & 164.9 \\
\hline 6 & 158.11 & 159.08 & 164.96 & 166.23 \\
\hline 6.5 & 159.29 & 160.27 & 166.17 & 167.45 \\
\hline 7 & 160.39 & 161.38 & 167.29 & 168.59 \\
\hline 7.5 & 161.41 & 162.41 & 168.33 & 169.64 \\
\hline 8 & 162.36 & 163.37 & 169.31 & 170.63 \\
\hline 8.5 & 163.26 & 164.28 & 170.23 & 171.56 \\
\hline 9 & 164.11 & 165.13 & 171.09 & 172.43 \\
\hline 9.5 & 164.91 & 165.94 & 171.91 & 173.26 \\
\hline 10 & 165.67 & 166.71 & 172.68 & 174.04 \\
\hline & & & & \\
\hline & & & & \\
\hline
\end{tabular}

\subsection{Data Analysis using RMSE}

RMSE (Root mean square error) statistic gives a quantitative measure of how close the predicted path loss values (COST 231 ) are to the measured path loss values. RMSE value closer to zero indicates a better fit. It is given as stated below

$R M S E=\sqrt{\sum_{i=1}^{k} \frac{\left[P L_{m}(d)-P L_{r}(d)\right]^{2}}{k}} \ldots$

Where PLm $(\mathrm{d})=$ measured pathloss $(\mathrm{dB}), \operatorname{PLr}(\mathrm{d})=$ calculated path loss $(\mathrm{dB})$ and $\mathrm{k}=20$ (number of measured data points).

Equation (5) above was applied to the numerical values of the measured path loss and the predicted pathloss(Cost231) to obtain the RMSEs for each environment under study as shown in TABLE 8 below.

Table 8. RMSEs of Calculated Pathloss and Measured pathloss

\begin{tabular}{|l|l|l|l|}
\hline Terrain & $\begin{array}{l}\text { 2G }-\mathbf{9 0 0} \\
\text { MHz }\end{array}$ & $\begin{array}{l}\text { 2G-1800 } \\
\text { MHz }\end{array}$ & $\begin{array}{l}\text { 3G-2100 } \\
\text { MHz }\end{array}$ \\
\hline NU & 3.23 & 3.33 & 5.08 \\
\hline SU & 1.74 & 3.38 & 2.99 \\
\hline UR & 3.88 & 6.15 & 5.70 \\
\hline DU & 7.11 & 6.49 & 9.24 \\
\hline
\end{tabular}

\subsection{Result Analysis}

The results of this study revealed that the Cost 231-Hata model showed a satisfactory performance in the chosen environments based on its RMSE values as shown in Table 8, and hence the following are our findings during the great research project:

a) Pathloss increases with distance between BTS and MS see figures 9, 10, 11 below and Table 11 for tabular categorization of distance and the pathloss rating.

b) Pathloss also increases as frequency increases; hence $3 \mathrm{G}$ network experience more pathloss than $2 \mathrm{G}$ networks. See figures 12, 13, 14 and 15 for proofs and Table 10 for frequency versus pathloss rating.

c) Pathloss, from our findings increases from NonUrban to Sub-Urban to Urban and Dense-Urban environments respectively. Check figures 9,10 and 11 below and Table 9 for Terrain against pathloss rating.

Table 9. Pathloss Comparison Based on Terrain

\begin{tabular}{|l|l|l|l|}
\hline \multirow{2}{*}{ SN } & \multicolumn{3}{|c|}{ Pathloss Comparison Based on Terrain } \\
& Enviference & \\
\cline { 2 - 4 } & Environment & Nomenclature & Rating \\
\hline 1 & Non- Urban & NU & Low \\
\hline 3 & Urban & SU - Urban & Medium \\
\hline 4 & Dense- Urban & DU & High \\
& & & $\begin{array}{l}\text { Relatively } \\
\text { High }\end{array}$ \\
\hline
\end{tabular}

Table 10. Pathloss Comparison Based on Frequency

\begin{tabular}{|l|l|l|l|}
\hline \multirow{2}{*}{ SN } & \multicolumn{3}{|c|}{ Pathloss Comparison Based on frequency of } \\
& operation \\
\cline { 2 - 4 } & Generation & Frequency (MHz) & Rating \\
\hline 1 & $2 \mathrm{G}$ & 900 & Low \\
\hline 2 & $2 \mathrm{G}$ & 1800 & Medium \\
\hline 3 & $3 \mathrm{G}$ & 2100 & $\begin{array}{l}\text { Relatively } \\
\text { High }\end{array}$ \\
\hline
\end{tabular}

Table 11. Pathloss Comparison Based on Distance

\begin{tabular}{|l|l|l|l|}
\hline \multirow{2}{*}{ SN } & \multicolumn{3}{|c|}{ Pathloss Comparison Based on Distance between } \\
& BTS and MS \\
\cline { 2 - 4 } & Distance Rating & $\begin{array}{l}\text { Distance in } \\
\text { Km }\end{array}$ & Rating \\
\hline 1 & Very Near & $0.5-2.0$ & Very Low \\
\hline 2 & Near & $2.5-4.0$ & Low \\
\hline 3 & Average & $4.5-6.0$ & Medium \\
\hline
\end{tabular}




\begin{tabular}{|l|l|l|l|}
\hline 4 & Far & $6.5-8.0$ & High \\
\hline 5 & Very Far & $8.5-10.0$ & Very High \\
\hline
\end{tabular}

MATLAB 2015 edition was used to plot the graphs of the measured and calculated paththloss values in all environments to give a pictorial analysis of our research work as we have in figures (9-15) below:

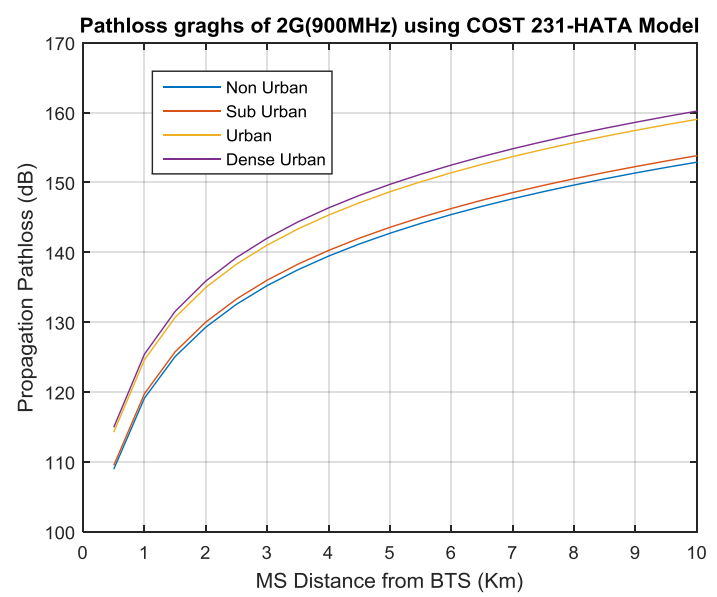

Fig 9: Pathloss comparison of 2G-900 MHz in different environs using COST 231-Hata Model

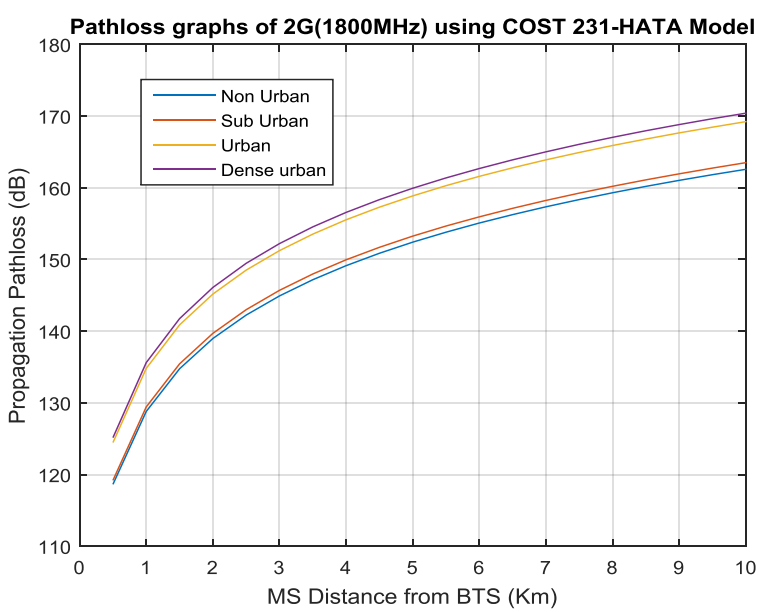

Fig 10: Pathloss comparison of 2G-1800 MHz in different environs using COST 231-Hata Model

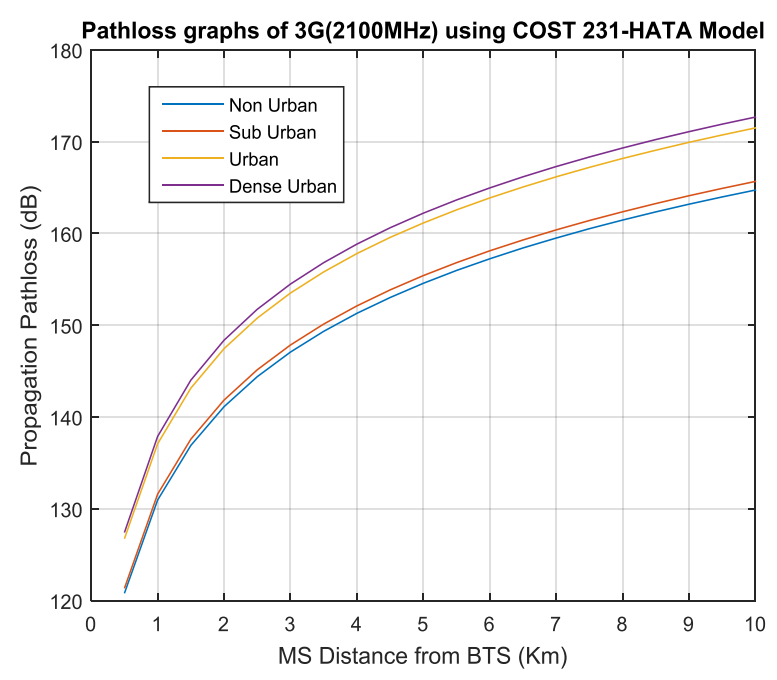

Fig 11: Pathloss comparison of 3G-2100 MHz in different environs using COST 231-Hata Model

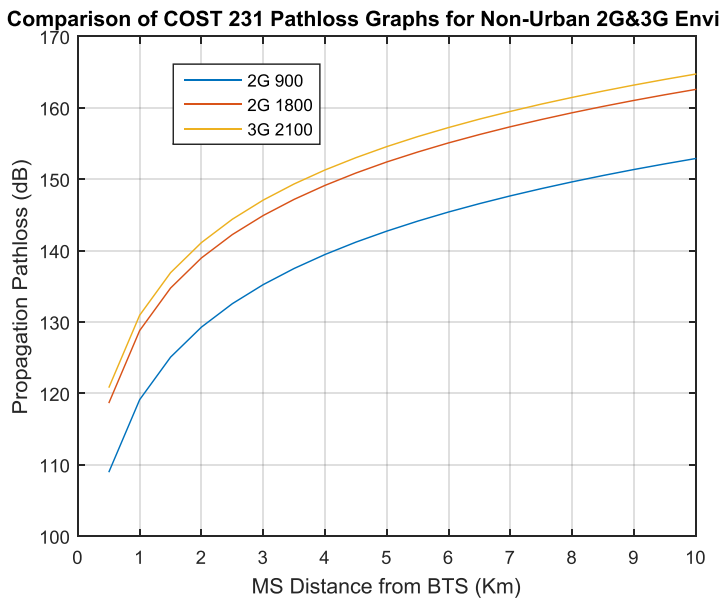

Fig 12: Pathloss comparison of $2 \mathrm{G} \& 3 \mathrm{G}$ frequencies in Non-Urban environs using COST 231-Hata Model

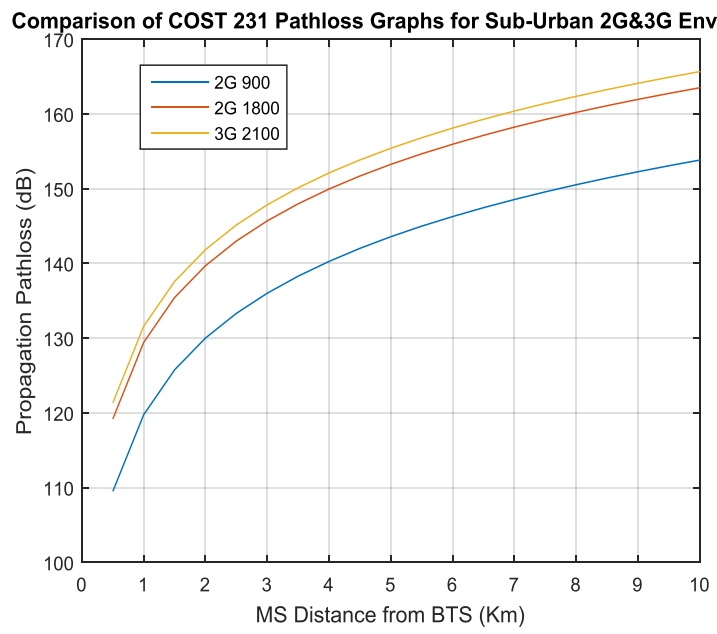

Fig 13: Pathloss comparison of $2 \mathrm{G} \& 3 \mathrm{G}$ frequencies in Sub-Urban environs using COST 231-Hata Model 


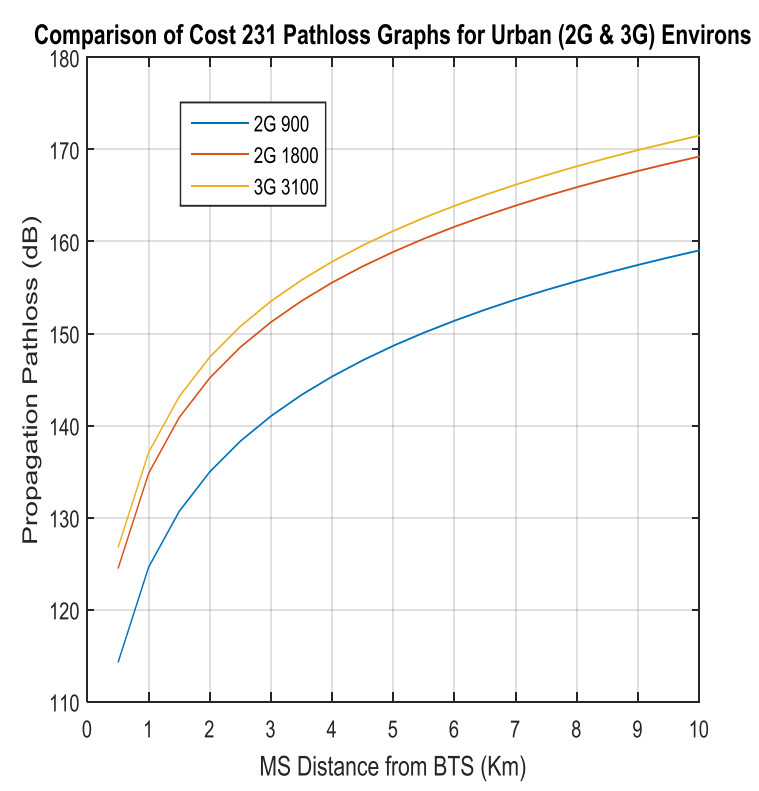

Fig 14: Pathloss comparison of $2 G \& 3 G$ frequencies in Urban environs using COST 231-Hata Model

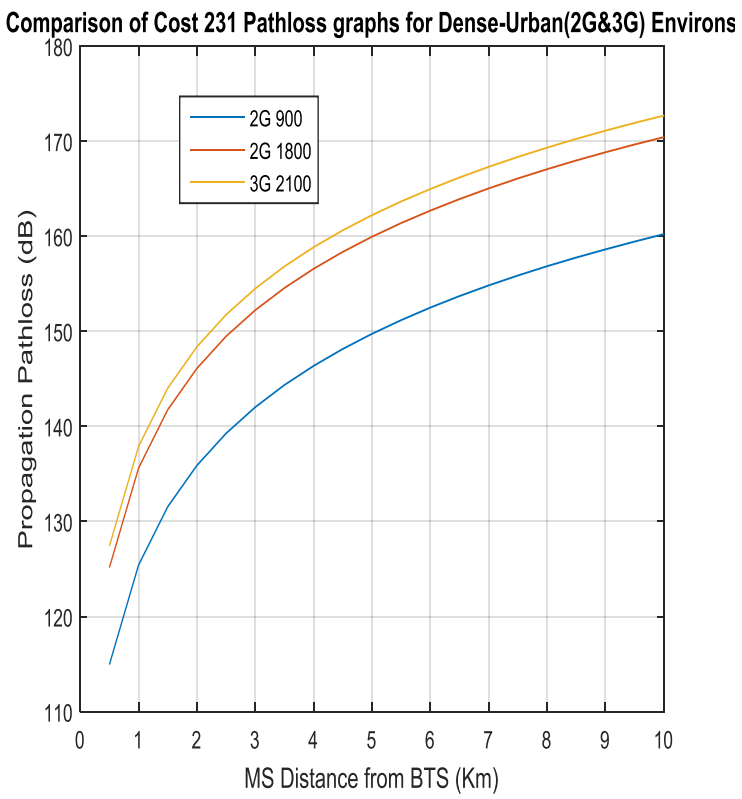

Fig 15: Pathloss comparison of $2 \mathrm{G} \& 3 \mathrm{G}$ frequencies in Dense-Urban environs using COST 231-Hata Model

\section{CONCLUSION}

The result of this research work helps users and researchers understand the G.S.M networks behavior based on parameters used for investigation. It also consolidate the usefulness of COST231-Hata model which showed a close agreement with the measured pathloss in terms of mean square error analysis, although this has been optimized for improved prediction purpose for operators in Lagos state or similar environments for pathloss prediction. ${ }^{[18]}$ However this research analysis can be used as a guide to aid network optimization process in the area of height of antennae which when increased reduces pathloss and appropriate Transmit power adjustment especially in dense-urban areas for improved performance for service providers in Lagos and other similar G.S.M environments.

\section{ACKNOWLEDGMENTS}

Wish to appreciate Dr. Femi Ipinnimo, Prof. Lawrence Oborkhale, the for their contribution to this study.

\section{REFERENCES}

[1] L. Akinyemi, N. Makanjuola, O. Shoewu, and F. Edeko, "Evaluation and Analysis of 3G Network in Lagos Metropolis, Nigeria," Science and Education, vol. 2, pp. 81-87, 2014.

[2] Danladi Ali, Natalia V. A, Measurement and Modeling of Path Loss for GSM Signal in a Sub Urban Environment over irregular terrain review. International Journal of Science and Research (IJSR) Volume 3 Issue 8, August 2014.

[3] S. Ajose and A.L. Imoize, "Propagation measurements and modelling at $1800 \mathrm{MHz}$ in Lagos Nigeria," International Journal of Wireless and Mobile Computing, vol. 6, pp. 165-174, 2013.

[4] Mustafa Bin Amar :Drive Test Overview

[5] Isabona Joseph1, Konyeha. C. C, Chinule. C. Bright, Isaiah Gregory Peter, Radio Field Strength Propagation Data and Pathloss calculation Methods in UMTS Network(2013)

[6] Mardeni, R. and Priya, T.S. "Optimized COST 231 Hata models for WiMAX pathloss prediction in suburban and open urban environments,".Modern Applied Science, vol. 4(9)- (2010)

[7] Goldsmith, A. "Wireless Communication." Cambridge University Press, New York. - (2005).

[8] Nochiri.Ifeoma.U, C.C Osuagwu, K.C. Okafor, Empirical Analysis on the GSM Network KPIs Using Real-Time Methodology for a Novel Network Integration Progress In Science and Engineering Research Journal (PISER) 13, Vol.02, Issue: 03/06 MayJune; Bimonthly International Journal.-(2014) pg: 92-107

[9] Prof. Theodore S. Rappaport, Wireless Communications Principles and Practice, $2^{\text {nd }}$ Edition, University of Texas, U.S.A-2002

[10] John S. Seybold, Wireless Communication Principles and practice, $2^{\text {nd }}$ Edition, U.S.A-(2005)

[11] Sylvain Ranvier, Path loss models S-72.333 Physical layer methods in wireless communication systems-(2014)

[12] A. Obot, O. Simeonb, and J. Afolayan, "Comparative analysis of path loss prediction models for urban macrocellular environments," Nigerian Journal of Technology, vol. 30, 2011

[13] Google.com (2016)

[14] Alatishe Adeyemi, Adu Oluwadamilola, Atayero Aderemi, Idachaba Francis, A Performance Review of the Different path Loss models for LTE Network Planning, World Congress on Engineering 2014 Vol I, WCE 2014, July 2 - 4, 2014, London, U.K.

[15] Syahfrizal Tahcfulloh, Eka Riskayadi Optimized Suitable Propagation Model for GSM 900Path Loss Prediction TELKOMNIKA Indonesian Journal of Electrical Engineering, Vol. 14, No. 1, April 2015, pp. 154 162, 
DOI: 10.11591/telkomnika.v14i1.7470

[16] A.U Usman, O.U Okereke, E.E. Omizegba Comparative analysis of pathloss model predictions with measurements taken for G.S.M 900/1800 MHZ networks in Bauchi suburbs of Nigeria. July 2012

[17] NT Surajudeen Bakinde, N Faruk, AA Ayeni, MY Muhammad, MI Gumel. Comparison of Propagation
Models for GSM 1800 and WCDMA Systems in Selected Urban Areas of Nigeria. International Journal of Applied Information Systems (IJAIS). 2012; 2(7): 6-13.

[18] O.O. Shoewu, N.O. Salau , A.O Ogunlewe, L.I Oborkharle Pathloss Measurement and Modeling For Lagos State G.S.M Environment- Review of Computer Engineering Research, 2016, 3(4): 69-81 